\title{
COMPACTAÇÃO DE SOLOS CULTIVADOS COM CANA-DE-AÇÚCAR: II - QUANTIFICAÇÃ̃O DAS RESTRIÇÕES ÀS FUNÇÕES EDÁFICAS DO SOLO EM DECORRÊNCIA DA COMPACTAÇÃO PREJUDICIAL
}

\author{
EDUARDO DA C. SEVERIANO ${ }^{1}$, GERALDO C. DE OLIVEIRA ${ }^{2}$, \\ MOACIR DE S. DIAS JÚNIOR ${ }^{3}$, MATHEUS B. DE CASTRO ${ }^{4}$, LUIZ F. C. DE OLIVEIRA ${ }^{5}$, \\ KATIA A. DE P. COSTA ${ }^{6}$
}

\begin{abstract}
RESUMO: A redução do período de entressafra canavieiro pode levar à disseminação da compactação do solo, em decorrência da colheita mecanizada da cana-de-açúcar na estação chuvosa. Dessa forma, faz-se necessário definir estratégias que visem a minimizar a esse processo. O presente estudo objetivou modelar o processo de compressão do solo com base na pressão crítica. Avaliou também os efeitos da colheita mecanizada da cana-de-açúcar em diferentes épocas da safra em um Latossolo Vermelho-Amarelo (LVA) e em um Cambissolo Háplico (CX). Determinaram-se em amostras indeformadas a macroporosidade do solo, a pressão de preconsolidação em diferentes conteúdos de água no solo e a densidade do solo. A partir da incorporação da densidade do solo crítica na modelagem do comportamento compressivo do solo, foi possível estimar a pressão crítica. A pressão crítica superestima a capacidade de suporte de carga dos solos. A colheita mecanizada da cana-de-açúcar, realizada na zona de friabilidade do solo, não provocou compactação prejudicial à cultura. A cana-de-açúcar pode ser colhida mecanicamente sem causar a degradação estrutural no LVA e no CX, respectivamente, sob conteúdos de água de até 0,16 e $0,21 \mathrm{~kg} \mathrm{~kg}^{-1}$.
\end{abstract}

PALAVRAS-CHAVE: Saccharum sp., degradação estrutural, pressão crítica, colheita mecanizada de cana-de-açúcar.

\section{COMPACTION OF SOILS CULTIVATED WITH SUGARCANE: II - QUANTIFICATION OF RESTRICTIONS TO SOIL EDAPHIC FUNCTIONS AS A RESULT OF HARMFUL COMPACTION}

\begin{abstract}
The reduction of a second growing season of sugarcane can lead to spread soil compaction, due to mechanical harvesting of sugarcane in the rainy season. Thus, it is necessary to define strategies to minimize this process. This study aimed to model the compressive behavior of the soil based on the critical pressure. The effects of the mechanized harvesting of sugarcane at different times of the crop were also assessed in a Red-Yellow (LVA) and in a Haplic Cambisol (CX). Soil macroporosity, preconsolidation pressure at different soil water contents and bulk density were determined in undisturbed soil samples. With the incorporation of the critical bulk density in modeling the soil compressive behavior, it was possible to estimate the critical pressure. The critical pressure overestimates the soil bearing capacity. The mechanized harvesting of sugarcane made in the friability zone did not cause harmful compaction to the crop. The sugarcane can be harvested mechanically without causing structural degradation in the LVA and CX, respectively, at soil water contents up to 0.16 and $0.21 \mathrm{~kg} \mathrm{~kg}^{-1}$.
\end{abstract}

KEYWORDS: Saccharum sp., structural degradation, critical pressure, mechanized harvesting of sugarcane.

\footnotetext{
${ }^{1}$ Eng $^{\mathrm{o}}$ Agrônomo, Instituto Federal de Educação, Ciência e Tecnologia Goiano, Câmpus Rio Verde - GO, Fone: (0XX64) 3620.5636, severianoec@yahoo.com.br. Bolsista de Desenvolvimento Científico Regional (FAPEG/CNPq).

${ }^{2}$ Eng $^{\mathrm{o}}$ Agrícola, Professor Adjunto, Departamento de Ciência do Solo (DCS), Universidade Federal de Lavras (UFLA), Lavras MG, geraldooliveira@dcs.ufla.br. Bolsista CAPES - Tutor PET Agronomia da UFLA.

${ }^{3}$ Eng $^{\mathrm{O}}$ Agrícola, Professor Associado, DCS/UFLA, msouzadj@dcs.ufla.br. Bolsista do CNPq.

${ }^{4}$ Eng ${ }^{\circ}$ Agrônomo, Mestrando em Fitotecnia, Departamento de Agricultura, DAG/UFLA, matheusbcastro87@ hotmail.com.

${ }^{5}$ Eng ${ }^{\mathrm{O}}$ Agrícola, Professor Associado, Departamento de Engenharia, DEG/UFLA. Bolsista do CNPq.

${ }^{6}$ Professora do Programa de Pós-Graduação em Produção Vegetal, Universidade de Rio Verde (FESURV), Rio Verde - GO.

Recebido pelo Conselho Editorial em: 17-4-2009
}

Aprovado pelo Conselho Editorial em: 12-12-2009 


\section{INTRODUÇÃO}

A pressão pelo uso de fontes de energia alternativa aos combustíveis fósseis, observada nas últimas décadas, vem fazendo com que os agrossistemas canavieiros sejam cada vez mais explorados com tecnologias baseadas no uso de máquinas agrícolas em todas as etapas do processo produtivo (PAULINO et al., 2004). Para tanto, faz-se a opção por veículos de maior capacidade unitária de carga, os quais, muitas vezes, trafegam sobre o solo em condições desfavoráveis em termos de conteúdo de água, tornando praticamente inevitável a ocorrência da compactação do solo (IAIA et al., 2006). Esse problema tem sido considerado um dos principais fatores de degradação da sua estrutura (MOSADDEGHI et al., 2007).

Nesse sentido, SOUZA et al. (2005), ao avaliarem o efeito de sistemas de manejo da cultura da cana-de-açúcar nos atributos físicos de um Latossolo Vermelho-Amarelo distrófico de textura média, constaram que a compactação do solo, em decorrência da colheita mecanizada dessa cultura, pode promover reduções superiores a $50 \%$ no volume de macroporos do solo. Por sua vez, tal alteração estrutural pode vir a comprometer a sustentabilidade dessa atividade agrícola, uma vez que essa classe de poros, em última análise, determina a taxa de movimentação de água no solo (MESQUITA \& MORAES, 2004), a recarga do lençol freático e, consequentemente, potencializam as perdas de solo e nutrientes por erosão e o assoreamento dos mananciais (IZIDORIO et al., 2005).

Considerando a tendência de amenização da estacionalidade da produção agrícola com a redução do período da entressafra da cultura, essa situação pode ser agravada pela colheita mecanizada durante a estação chuvosa na região Centro-Sul do Brasil (SEVERIANO et al., 2010). Dessa forma, monitorar a compactação do solo é de fundamental importância na manutenção da longevidade dos canaviais (BRAUNBECK \& OLIVEIRA, 2006), o que significa controlar os níveis de pressão aplicados pelos maquinários ou definir estratégias de manejo baseadas na predição dos impactos das operações sobre a estrutura do solo, visando a auxiliar a tomada de decisões em torno do momento adequado à realização das operações mecanizadas.

Na predição da capacidade de suporte de carga do solo, a pressão de preconsolidação tem sido muito utilizada (PENG et al., 2004; ARAÚJO JÚNIOR et al., 2008; DIAS JÚNIOR et al., 2008). Esse atributo quantifica o histórico de pressões que o solo já sofreu e representa a máxima pressão a ser aplicada no solo antes que a compactação adicional ocorra (DIAS JÚNIOR \& PIERCE, 1996).

Pesquisas realizadas com esse atributo têm revelado valores em torno de $10 \%$ no incremento da densidade do solo (KONDO \& DIAS JÚNIOR, 1999) ou na redução da porosidade total (OLIVEIRA et al., 2003) como indicativos de ocorrência de compactação adicional. Sugere-se, entretanto, que seu uso seja associado a índices restritivos, uma vez que a compactação adicional pode resultar em benefícios em termos do aumento na retenção de água (RESENDE et al., 2007), desde que ocorra em pequena intensidade, mas também pode ser determinadora de processos erosivos e mesmo limitante aos processos produtivos (REICHERT et al., 2007; SUZUKI et al., 2007), caso ocorra em níveis mais elevados.

Modelando a compactação do solo, IMHOFF et al. (2001) propuseram o conceito da pressão crítica para o crescimento das plantas, considerada a máxima pressão a ser aplicada no solo sem promover a degradação da qualidade estrutural do solo para o crescimento das plantas. Essa pressão é estimada em função do conteúdo de água no solo e da densidade de solo associada às condições restritivas para o crescimento radicular.

Para a estimativa desse atributo, os autores basearam-se em valores restritivos referentes ao parâmetro denominado intervalo hídrico ótimo, por ser esse o indicador biofísico do solo que melhor se correlaciona à produção vegetal (TORMENA et al., 2007). Contudo, segundo SEVERIANO et al. (2008), nessa condição estrutural, a porosidade drenável já pode estar sendo comprometida. Assim, os autores sugerem que a definição da pressão crítica seja baseada na redução da macroporosidade ao valor de $0,10 \mathrm{dm}^{3} \mathrm{dm}^{-3}$, por estar associada a condições estruturais 
do solo favoráveis à obtenção de produções satisfatórias, sem comprometer o seu papel ecológico na paisagem.

Nesse contexto, o presente trabalho objetivou modelar o processo de compressão do solo com base na pressão crítica, além de avaliar o efeito das operações de colheita mecanizada da cana-de-açúcar em diferentes épocas da safra em um Latossolo Vermelho-Amarelo e em um Cambissolo Háplico, no município de Goianésia - GO.

\section{MATERIAL E MÉTODOS}

O estudo foi conduzido nas áreas experimentais pertencentes à empresa Jalles Machado S.A., localizada no município de Goianésia - GO, a $15^{\circ} 10^{\prime} \mathrm{S}$ de latitude e $49^{\circ} 15^{\prime} \mathrm{W}$ de longitude e $640 \mathrm{~m}$ de altitude. O clima é classificado, segundo Köeppen, como tropical de savana, quente e úmido, com inverno seco e verão chuvoso (Aw), e média pluvial anual de $1.500 \mathrm{~mm}$.

Na condução deste estudo, selecionaram-se áreas experimentais em um Latossolo Vermelho-Amarelo (LVA) e em um Cambissolo Háplico (CX) (EMBRAPA, 2006). Os tratamentos consistiram na colheita mecanizada da cana-de-açúcar, realizada nos meses de novembro de 2005, março e agosto de 2006, e na colheita manual sem tráfego, em março de 2006, por ser a época de maior intensidade pluvial, onde suas características, bem como o histórico de uso das áreas, tamanho das parcelas, conteúdo de água no solo durante a colheita, a forma de amostragem e os procedimentos analíticos foram descritos em (SEVERIANO et al., 2010).

Na obtenção dos dados experimentais, foram determinadas em 288 amostras indeformadas (6 amostras x 12 parcelas x 2 solos x 2 amostragens de solo: antes e após as operações de colheita da cana-de-açúcar), a macroporosidade do solo, seguindo a metodologia proposta por EMBRAPA (1997); a pressão de preconsolidação $\left(\sigma_{\mathrm{p}}\right)$, a partir do ensaio de compressibilidade em diferentes conteúdos de água no solo (U) conforme DIAS JÚNIOR \& PIERCE (1995), e a densidade do solo (Ds), após secagem das amostras utilizadas nas determinações dos parâmetros acima, em estufa a $105^{\circ} \mathrm{C}$, por 48 horas, segundo EMBRAPA (1997).

A pressão de preconsolidação foi ajustada ao modelo não linear proposto por BUSSCHER (1990) e que tem sido aceita para uso em dados de resistência do solo à penetração em função do conteúdo de água no solo (U) e da Ds:

$$
\sigma_{\mathrm{p}}=\mathrm{aU}^{\mathrm{b}} \mathrm{Ds}_{\mathrm{i}}{ }^{\mathrm{c}}
$$

em que,

$\sigma_{\mathrm{p} \text { - }}$ pressão de preconsolidação, $\mathrm{kPa}$;

U - conteúdo de água no solo, $\mathrm{kg} \mathrm{kg}^{-1}$;

Ds - densidade do solo, e

"a", "b" e "c" - coeficientes de ajuste da equação de regressão.

Apesar da carência de aplicação do referido ajuste em dados de pressão de preconsolidação, em função da Ds e U, a tentativa de uso dessa proposta baseou-se na forte relação entre $\sigma_{\mathrm{p}}$ e RP (MOSADDEGHI et al., 2003; DIAS JÚNIOR et al., 2004; LIMA et al., 2006), indicando comportamento físico semelhante das duas variáveis. A densidade do solo crítica (Dsc $\mathrm{MAC}_{\text {) foi }}$ considerada como sendo a correspondente à macroporosidade de $0,10 \mathrm{dm}^{3} \mathrm{dm}^{-3}$, conforme as considerações realizadas por SEVERIANO et al (2008), sendo essa obtida pelo ajuste linear entre essas variáveis. A pressão crítica $\left(\sigma_{\mathrm{cr}}\right)$ foi determinada tendo como parâmetros de modelagem a $\mathrm{Dsc}_{\mathrm{MAC}}$ e o conteúdo de água no solo ajustado na amostra para o ensaio de compressibilidade.

Os modelos de capacidade de suporte de carga dos solos foram obtidos ajustando-se os valores de pressão de preconsolidação $\left(\sigma_{\mathrm{p}}\right)$ e os valores de pressão crítica $\left(\sigma_{\mathrm{cr}}\right)$ em função do conteúdo de água no solo, conforme DIAS JÚNIOR (1994). As comparações entre elas foram realizadas conforme SNEDECOR \& COCHRAN (1989). Determinou-se o intervalo de confiança, a 95\%, dos modelos de capacidade de suporte de carga, e as três regiões propostas por DIAS JÚNIOR 
et al. (2005) foram utilizadas no monitoramento da compactação dos solos em decorrência das operações de colheita da cana-de-açúcar.

\section{RESULTADOS E DISCUSSÃO}

As eqs.(2) e (3) demonstram com elevado poder de predição, mas com ajuste não linear, que a pressão de preconsolidação $\left(\sigma_{\mathrm{p}}\right)$ decresce com o incremento de água no solo (U) e aumenta com a densidade do solo (Ds).

$$
\begin{aligned}
& \text { LVA : } \sigma_{\mathrm{p}}=2,64 \mathrm{U}^{-1,24} \mathrm{Ds}_{\mathrm{i}}^{4,93} ; \mathrm{R}^{2}=0,83 * * \\
& \mathrm{CX}: \sigma_{\mathrm{p}}=20,77 \mathrm{U}^{-0,91} \mathrm{Ds}_{\mathrm{i}}^{3,43} ; \mathrm{R}^{2}=0,72 * *
\end{aligned}
$$

Para a estimativa da pressão de preconsolidação, IMHOFF et al. (2001) propuseram um ajuste linear da $\sigma_{\mathrm{p}}$ em função de U e Ds. Entretanto, a variação do conteúdo de água no solo, no referido trabalho, foi do ponto de murcha permanente até próximo da capacidade de campo, demonstrando que, nesse intervalo de umidade, a $\sigma_{\mathrm{p}}$ decresce linearmente com o incremento de U. Em contrapartida, quando o solo se encontra sob conteúdos superiores à capacidade de campo, essa relação é exponencial (DIAS JÚNIOR, 1994), o que justifica o uso do ajuste não linear proposto por BUSSCHER (1990). Como já salientado, apesar da carência de aplicação do referido ajuste na modelagem do comportamento compressivo do solo, a aceitação dessa proposta é baseada na significância das regressões $(\mathrm{F}=167,09 ; \mathrm{P}<0,001$ e $\mathrm{F}=188,87 ; \mathrm{P}<0,001$ para LVA e CX, respectivamente).

Embora a densidade do solo seja a medida quantitativa mais direta no diagnóstico da compactação, CAMARGO \& ALLEONI (1997) ressalvam a influência exercida pela textura e estrutura do solo nesse atributo, limitando a escolha de um valor absoluto de referência. Por outro lado, com base no valor de macroporosidade, é possível estimar o valor crítico de Ds, associado com condições estruturais desfavoráveis ao pleno desenvolvimento das culturas e ao papel ecológico dos solos na paisagem (REICHERT et al., 2007), relacionado à infiltração de água e recarga do lençol freático, tendo em vista a forte correlação existente entre esses atributos (OLIVEIRA et al., 2007). Dessa forma, para o valor de macroporosidade de $0,10 \mathrm{dm}^{3} \mathrm{dm}^{-3}$, são sugeridos os valores correspondentes de densidade crítica (Dsc $\mathrm{MAC}_{\mathrm{C}}$ ), para o CX e LVA, de 1,44 e $1,59 \mathrm{~kg} \mathrm{dm}^{-3}$, respectivamente (Figura 1).

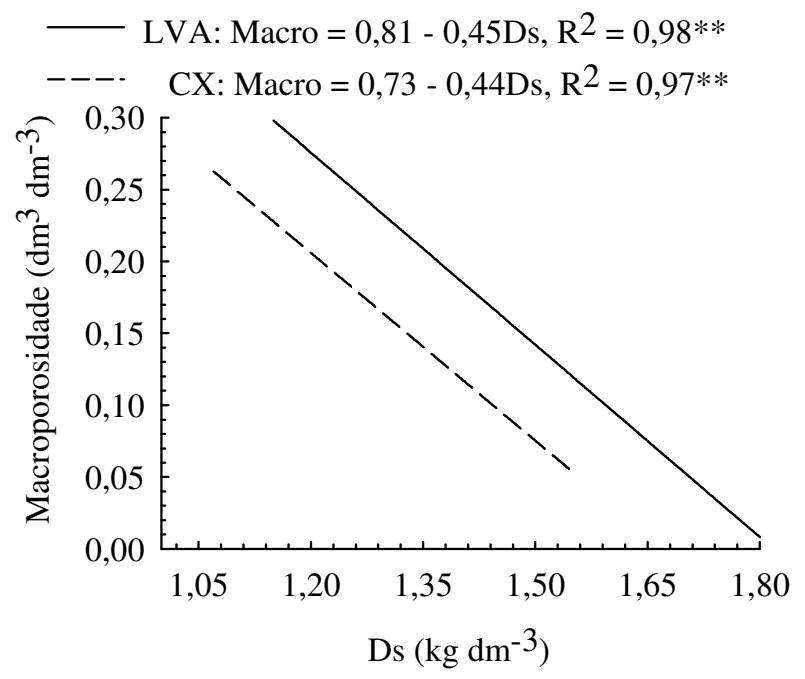

FIGURA 1. Macroporosidade do solo em função da densidade do solo, de um Latossolo Vermelho-Amarelo (LVA) e um Cambissolo Háplico (CX), cultivados com cana-de-açúcar. Macroporosity as a function of soil bulk density, of a Red-Yellow Latosol (LVA) and a Haplic Cambisol (CX), cultivated with sugarcane. 


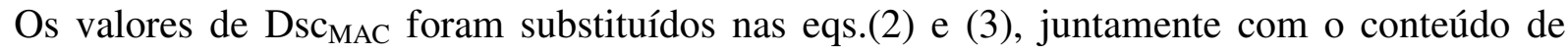
água no solo, ajustado na amostra durante a realização do ensaio de compressibilidade para a estimativa da pressão crítica $\left(\sigma_{\mathrm{cr}}\right)$, sendo essa considerada neste trabalho como sendo a máxima pressão a ser aplicada no solo sem que a provável ocorrência de compactação venha limitar as suas funções edáficas. Relacionando a pressão crítica $\left(\sigma_{\mathrm{cr}}\right)$ com a pressão de preconsolidação $\left(\sigma_{\mathrm{p}}\right)$ obtida em laboratório (Figura 2), observa-se que os valores estimados foram sempre superiores aos reais, conforme indicado pela linha 1:1. A discrepância entre as retas indica que não houve um ajuste perfeito na predição (ZHUANG et al., 2001).

Contudo, ressalta-se a $\sigma_{\mathrm{cr}}$ é estimada com base na Dsc $\mathrm{MAC}_{\mathrm{AC}}$, enquanto a $\sigma_{\mathrm{p}}$ corresponde à estrutura do solo inicial, sem qualquer compactação adicional. Conforme apontado por (SEVERIANO et al., 2010), observa-se que a densidade inicial dos dois solos (Dsi) foi inferior à Dsc $_{\text {MAC. }}$ Esses resultados indicam que, nessas condições, a pressão crítica pode superestimar a pressão de preconsolidação.

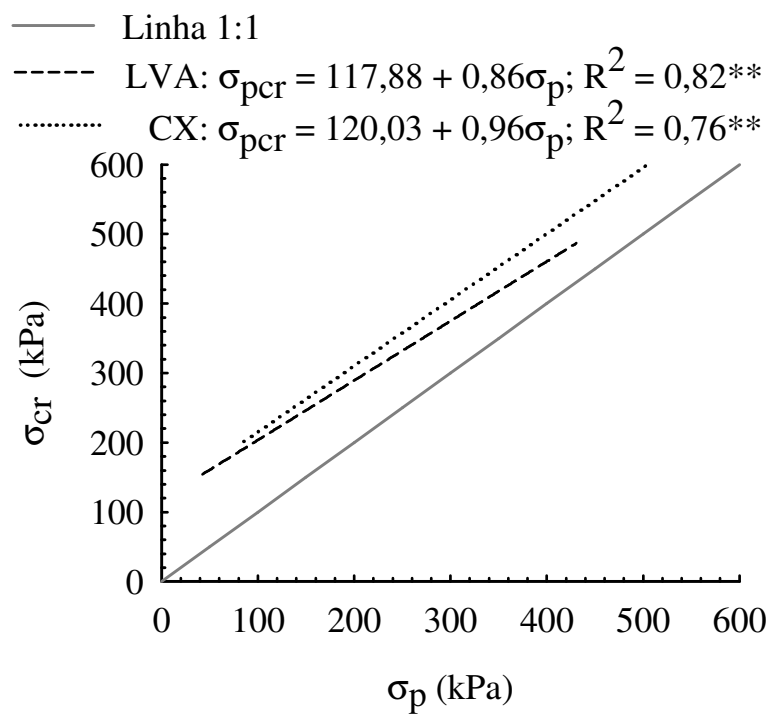

FIGURA 2. Representação dos valores de pressão crítica $\left(\sigma_{c r}\right)$ preditos pelas eqs.(2) e (3), em relação aos valores de pressão de preconsolidação $\left(\sigma_{\mathrm{p}}\right)$ determinados, do Latossolo Vermelho-Amarelo (LVA) e do Cambissolo Háplico (CX), cultivados com cana-de-açúcar. Representation of the critical pressure values $\left(\sigma_{\mathrm{cr}}\right)$ predicted by the equations 2 and 3 , in relation to the determined preconsolidation pressure values $\left(\sigma_{p}\right)$ of the Red-Yellow Latosol (LVA) and the Haplic Cambisol (CX), cultivated with sugarcane.

Portanto, o uso da pressão crítica assume a possibilidade de ocorrência de compactação adicional, desde que essa não seja suficientemente elevada para promover redução da macroporosidade a valores inferiores a $0,10 \mathrm{dm}^{3} \mathrm{dm}^{-3}$. Esses resultados corroboram OLIVEIRA et al. (2003), que encontraram valores de pressão a ser aplicada para se atingirem níveis considerados críticos de porosidade superiores à pressão de preconsolidação.

Ao se comparar o comportamento compressivo do solo com base nas pressões de preconsolidação $\left(\sigma_{\mathrm{p}}\right)$ e de preconsolidação crítica $\left(\sigma_{\mathrm{cr}}\right)$, observa-se, na Figura 3 , o aumento da capacidade de suporte de carga do solo quando esse já se encontra na condição limítrofe às funções edáficas (não homogêneo para as regressões $(\mathrm{NH}) ; \mathrm{F}=20,85 ; \mathrm{p}<0,001$ para o coeficiente linear e $\mathrm{F}=167,09 ; \mathrm{p}<0,001$ para o coeficiente angular para o LVA e $\mathrm{NH}, \mathrm{F}=6,97$ e 147,01; $\mathrm{p}<0,001$, respectivamente, para o CX, segundo SNEDECOR \& COCHRAN (1989).

Esse comportamento deve-se ao aumento do número de pontos de contato entre as partículas, promovido pela maior densidade do solo (SILVA et al., 2002), gerando maior atrito interno no solo, o que leva ao aumento dos valores de pressão de preconsolidação (SEVERIANO et al., 2010). 
(A)

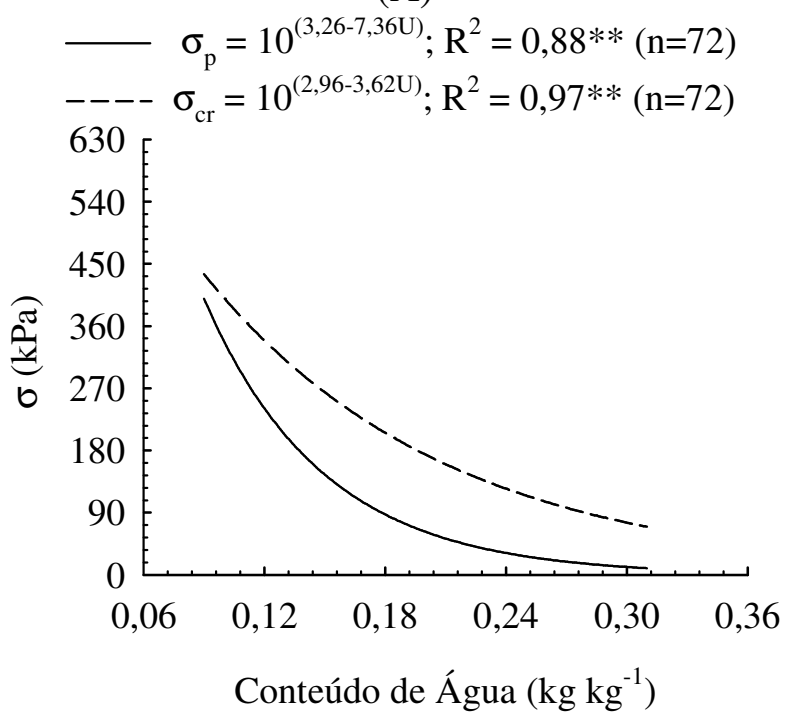

(B)

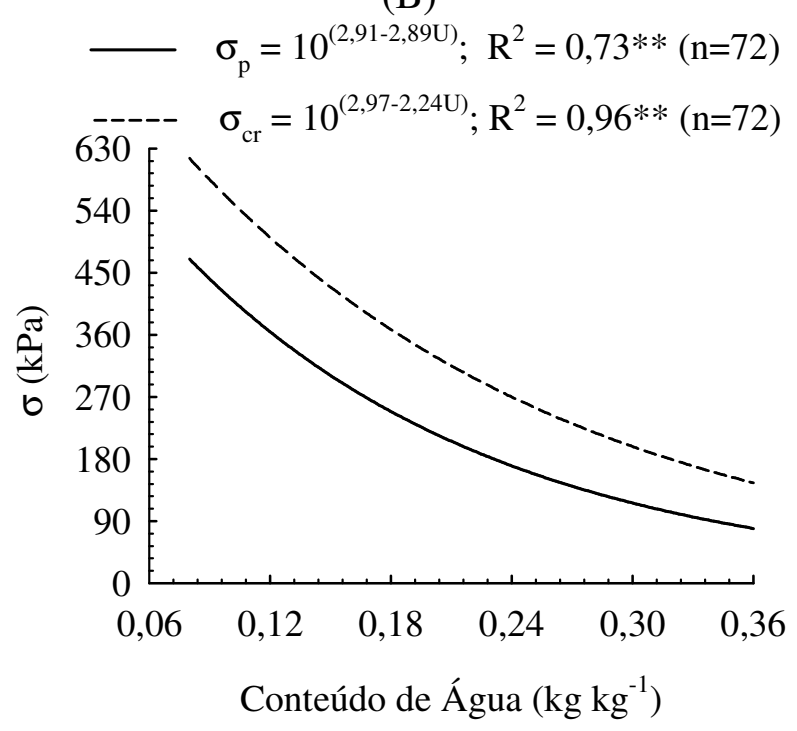

FIGURA 3. Modelos de capacidade de suporte de carga do solo com base na pressão de preconsolidação $\left(\sigma_{\mathrm{p}}\right)$ e na pressão crítica $\left(\sigma_{\mathrm{cr}}\right)$ de um $(\mathrm{A})$ Latossolo Vermelho-Amarelo (LVA) e de um (B) Cambissolo Háplico (CX), cultivados com cana-de-açúcar, na profundidade de $0-5 \mathrm{~cm}$. Soil bearing capacity models based on the preconsolidation pressure $\left(\sigma_{\mathrm{p}}\right)$ and the critical pressure $\left(\sigma_{\mathrm{cr}}\right)$, of a (A) Red-Yellow Latosol (LVA) and a (B) Haplic Cambisol (CX), cultivated with sugarcane, at 0-5 cm depth.

Para a avaliação dos efeitos das operações mecanizadas de colheita da cana-de-açúcar, os modelos de capacidade de suporte de carga do solo, com base na pressão crítica, foram divididos em três regiões e apresentados na Figura 4.

Como essa pressão leva em consideração as condições relacionadas à degradação estrutural e o desenvolvimento satisfatório das plantas, e, admitindo que na sua modelagem, haja a possibilidade de ocorrência de compactação adicional do solo, sem ocasionar, entretanto, prejuízos significativos às suas funções ecológicas na paisagem, na quantificação dos efeitos avaliados por esse parâmetro, faz-se necessário adaptar os critérios de avaliação das alterações estruturais do solo decorrente do tráfego de máquinas propostos por DIAS JÚNIOR et al. (2005) e utilizados em (SEVERIANO et al., 2010).

Dentre as modificações, sugere-se que os valores de pressão de preconsolidação determinados após o tráfego que se localizarem na região "a" sejam considerados como limitante às funções edáficas do solo; na região "b" sejam considerados sem compactação crítica, embora haja possibilidade de ocorrência de compactação adicional, e a região "c" como sendo sem compactação.

Considerando essa possibilidade de haver compactação adicional entre o intervalo de confiança, porém, sem que essa venha a limitar a funcionalidade do sistema solo, a redistribuição dos valores de pressão de preconsolidação avaliados após as operações de colheita da cana-de-açúcar demonstra que parte dos valores presentes na região "a" dos modelos de capacidade de suporte de carga do solo com base na pressão de preconsolidação (SEVERIANO et al., 2010) migrou para a região "b" dos modelos baseados na pressão crítica, e dessa para a "c" (Tabela 1).

Fica evidenciado, na Tabela 1, que apesar da ocorrência da compactação adicional, estando o solo na zona de friabilidade no momento das operações mecanizadas (conteúdo de água referente às colheitas em novembro de 2005, segundo SEVERIANO et al., 2010), essa não é considerada prejudicial, uma vez que as alterações estruturais nos solos, provavelmente, não foram suficientes para limitar o desenvolvimento da soqueira da cana-de-açúcar, além de favorecer relativa drenagem interna do solo (REICHERT et al., 2007). 
(A)

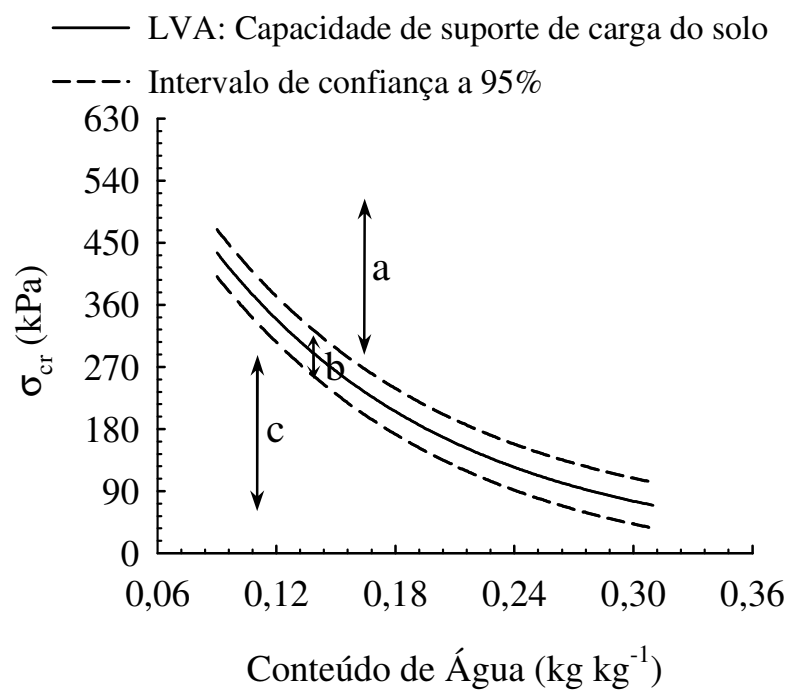

(B)

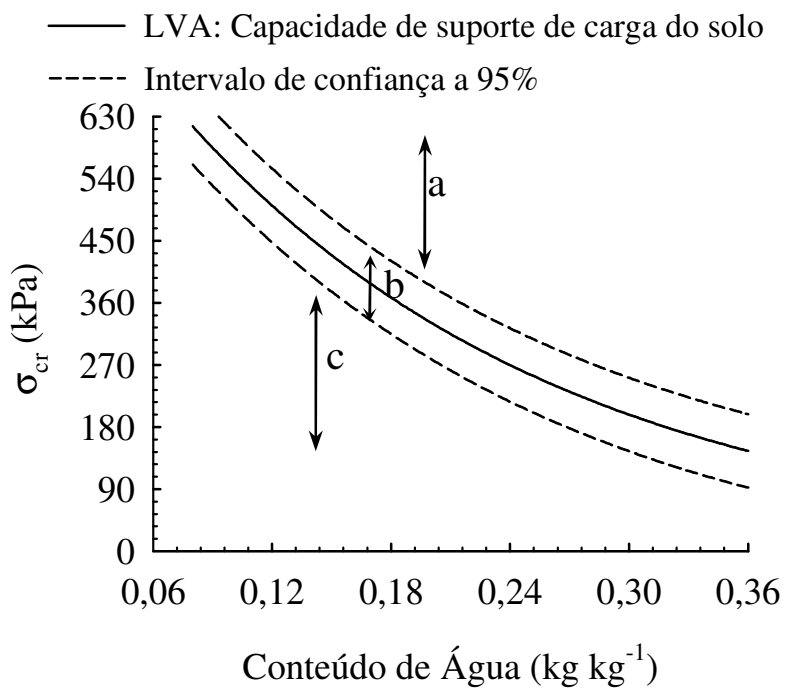

FIGURA 4. Modelos de capacidade de suporte de carga com base na pressão crítica ( $\left.\sigma_{\mathrm{cr}}\right)$ de um (A) Latossolo Vermelho-Amarelo (LVA) e de um (B) Cambissolo Háplico (CX), e critérios utilizados para analisar os efeitos das operações de colheita da cana-de-açúcar. "a": Região limitante às funções edáficas do solo, "b": sem compactação crítica e "c": sem compactação. Soil bearing capacity models based on the critical pressure $\left(\sigma_{\mathrm{cr}}\right)$ of a Red-Yellow Latosol (LVA) and a Haplic Cambisol (CX), and criteria used to assess the effects of the harvesting operations of sugarcane. "a": limiting region to soil edafics functions, "b": with no critical compaction and "c": with no soil compaction.

TABELA 1. Classificação das amostras do solo de acordo com a Figura 4, usando os valores de pressão $(\sigma)$ determinados depois de operações de colheita da cana-de-açúcar, em um Latossolo Vermelho-Amarelo (LVA) e em um Cambissolo Háplico (CX), na profundidade de $0-5 \mathrm{~cm}$. Classification of the soil samples according to Figure 4, using the pressure values $(\sigma)$ determined after harvesting operations of sugarcane, in a Red-Yellow Latosol (LVA) and a Haplic Cambisol (CX), at 0-5 cm depth.

\begin{tabular}{|c|c|c|c|c|c|c|}
\hline \multirow{3}{*}{ Região } & \multicolumn{3}{|c|}{ Mecanizada } & \multicolumn{3}{|c|}{ Mecanizada } \\
\hline & $11 / 2005$ & $3 / 2006$ & $8 / 2006$ & $11 / 2005$ & $3 / 2006$ & $8 / 2006$ \\
\hline & \multicolumn{3}{|c|}{-} & \multicolumn{3}{|c|}{ 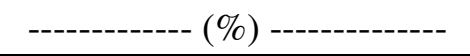 } \\
\hline & \multicolumn{3}{|c|}{------------------- LVA ------------------- } & \multirow[b]{2}{*}{0} & \multirow[b]{2}{*}{100} & \multirow[b]{2}{*}{0} \\
\hline a. Limitante às funções edáficas & & $265^{2}$ & - & & & \\
\hline b. Sem compactação crítica & $241^{5}$ & - & $428^{4}$ & 56 & 0 & 22 \\
\hline c. Sem compactação & $137^{6}$ & - & $253^{3}$ & 44 & 0 & 78 \\
\hline \multicolumn{7}{|c|}{ 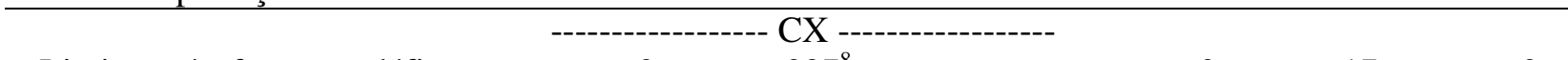 } \\
\hline a. Limitante às funções edáficas & 0 & $327^{8}$ & - & 0 & 17 & 0 \\
\hline b. Sem compactação crítica & $305^{3}$ & $258^{7}$ & $379^{6}$ & 78 & 83 & 44 \\
\hline c. Sem compactação & $235^{4}$ & - & $297^{5}$ & 22 & 0 & 56 \\
\hline
\end{tabular}

Apesar dos maiores conteúdos de água no CX em todas as épocas de colheita (SEVERIANO et al., 2010), os impactos das operações de colheita mecanizada nesse solo foram sempre inferiores aos observadas no LVA. Na época de maior intensidade pluvial da região (3/2006), a compactação nesse último solo, além de adicional, foi também crítica às funções edáficas em $100 \%$ das amostras coletadas após o tráfego do maquinário. Para o CX, a limitação atingiu apenas $17 \%$ dos pontos 
amostrados (Tabela 1). Dessa forma, a definição da época de colheita com o auxílio da pressão crítica $\left(\sigma_{\mathrm{cr}}\right)$ possibilita a realização das operações mecanizadas sob conteúdos de água no solo superiores àqueles definidos pela pressão de preconsolidação $\left(\sigma_{p}\right)$, dando maior flexibilidade no calendário da agroindústria canavieira e na tomada de decisões relacionadas à redução do período de entressafra.

\section{CONCLUSÕES}

A incorporação da densidade do solo na estimativa da pressão de preconsolidação permite a predição da pressão crítica.

Os valores de pressão crítica superaram os valores de pressão de preconsolidação dos solos estudados

Sugere-se o uso conjunto dos valores de pressão de preconsolidação e de pressão crítica para melhor entendimento de compactação adicional e compactação prejudicial.

A colheita mecanizada da cana-de-açúcar, realizada na zona de friabilidade do solo, não provocou compactação prejudicial ao desenvolvimento da soqueira da cana-de-açúcar.

A colheita mecanizada da cana-de-açúcar na área do Latossolo Vermelho-Amarelo estudado pode ser realizada quando o mesmo se encontrar com conteúdos de água de até $0,16 \mathrm{~kg} \mathrm{~kg}^{1}$, enquanto no Cambissolo Háplico o conteúdo de água no momento da colheita pode chegar a $0,21 \mathrm{~kg} \mathrm{~kg}^{-1}$.

\section{AGRADECIMENTOS}

Ao Conselho Nacional de Desenvolvimento Científico e Tecnológico (CNPq) e à empresa Jalles Machado S.A., pelo financiamento desta pesquisa.

\section{REFERÊNCIAS}

ARAÚJO JÚNIOR, C.F.; DIAS JÚNIOR, M.S.; GUIMARÃES, P.T.G.; PIRES, B.S. Resistência à compactação de um Latossolo cultivado com cafeeiro, sob diferentes sistemas de manejo de plantas invasoras. Revista Brasileira de Ciência do Solo, Viçosa - MG, v.32, n.1, p.23-32, 2008.

BRAUNBECK, O.A.; OLIVEIRA, J.T.A. Colheita de cana-de-açúcar com auxílio mecânico. Engenharia Agrícola, Jaboticabal, v.26, n.1, p.300-308, 2006.

BUSSCHER, W.J. Adjustment of flat-tipped penetrometer resistance data to common water content. Transactions of the ASAE, St. Joseph, v.3, n.2, p.519-524, 1990.

CAMARGO, O.A.; ALLEONI, L.R.F. Compactação do solo e o desenvolvimento de plantas. Piracicaba: Escola Superior de Agricultura "Luiz de Queiroz", 1997. 132 p.

DIAS JÚNIOR, M.S. Compression of three soils under long-term tillage and wheel traffic. 1994. 114 f. Thesis (Ph.D. in Crop and Soil Science) - Michigan State University, East Lansing, 1994.

DIAS JÚNIOR, M.S.; LEITE, F.P.; LASMAR JÚNIOR, E.; ARAÚJO JÚNIOR, C.F. Traffic effects on the soil preconsolidation pressure due to eucalyptus harvest operations. Scientia Agrícola, Piracicaba, v.62, n.3, p.248-255, 2005.

DIAS JÚNIOR, M.S.; PIERCE, F.J. A simple procedure for estimating preconsolidation pressure from soil compression curves. Soil Technology, Lansing, v.8, n.2, p.139-151, 1995.

DIAS JÚNIOR, M.S.; PIERCE, F.J. O processo de compactação do solo e sua modelagem. Revista Brasileira de Ciência do Solo, Viçosa - MG, v.20, n.2, p.175-182, 1996.

DIAS JÚNIOR, M.S.; SILVA, A.R.; FONSECA, S.; LEITE, F.P. Método alternativo de avaliação da pressão de preconsolidação por meio de um penetrômetro. Revista Brasileira de Ciência do Solo, Viçosa - MG, v.28, n.5, p.805-810, 2004. 
DIAS JÚNIOR, M.S.; SILVA, S.R.; SANTOS, N.S.; ARAÚJO JÚNIOR, C.F. Assessment of the soil compaction of two ultisols caused by logging operations. Revista Brasileira de Ciência do Solo, Viçosa - MG, v.36, n.6, p.2.245-2.253, 2008.

EMBRAPA. EMPRESA BRASILEIRA DE PESQUISA AGROPECUÁRIA. Manual de métodos de análises de solo. 2.ed. Rio de Janeiro: Ministério da Agricultura e do Abastecimento, 1997. $212 \mathrm{p}$.

EMBRAPA. EMPRESA BRASILEIRA DE PESQUISA AGROPECUÁRIA. Sistema brasileiro de classificação de solos. 2.ed. Rio de Janeiro: Ministério da Agricultura e do Abastecimento, 2006. $306 \mathrm{p}$.

IAIA, A.M.; MAIA, J.C.S.; KIM, M.E. Uso do penetrômetro eletrônico na avaliação da resistência do solo cultivado com cana-de-açúcar. Revista Brasileira de Engenharia Agrícola e Ambiental, Campina Grande, v.10, n.2, p.523-530, 2006.

IMHOFF, S.; SILVA, A.P.; DIAS JÚNIOR, M.S.; TORMENA, C.A. Quantificação de pressões críticas para o crescimento das plantas. Revista Brasileira de Ciência do Solo, Viçosa - MG, v.25, n.1, p.11-18, 2001.

IZIDORIO, R.; MARTINS FILHO, M.V.; MARQUES JÚNIOR, J.; SOUZA, Z.M.; PEREIRA, G.T. Perdas de nutrientes por erosão e sua distribuição espacial em área sob cana-de-açúcar. Engenharia Agrícola, Jaboticabal, v.25, n.3, p.660-670, 2005.

KONDO, M.K.; DIAS JÚNIOR, M.S. Compressibilidade de três latossolos em função da umidade e uso. Revista Brasileira de Ciência do Solo, Viçosa - MG, v.23, n.2, p.211-218, 1999.

LIMA, C.L.R.; SILVA, A.P.; IMHOFF, S.; LEÃO, T.P. Estimativa da capacidade de suporte de carga do solo a partir da avaliação da resistência à penetração. Revista Brasileira de Ciência do Solo, Viçosa - MG, v.30, n.2, p.217-233, 2006.

MESQUITA, M.G.B.F.; MORAES, S.O. A dependência entre a condutividade hidráulica saturada e atributos físicos do solo. Ciência Rural, Santa Maria, v.34, n.3, p.963-969, 2004.

MOSADDEGHI, M.R.; HEMMAT, A.; HAJABBASI, M.A.; ALEXANDROU, A. Precompression stress and its relation with the physical and mechanical properties of a structurally unstable soil in Central Iran. Soil \& Tillage Research, Amsterdam, v.70, n.1, p.53-64, 2003.

MOSADDEGHI, M.R.; KOOLEN, A.J.; HEMMAT, A.; HAJABBASI, M.A.; LERINK, P. Comparisons or different procedures of pre-compactation stress determination on weakly structure soils. Journal of Terramechanics, London, v.44, n.1, p.53-63, 2007.

OLIVEIRA, G.C.; DIAS JÚNIOR, M.S.; RESCK, D.V.S.; CURI, N. Compressibilidade de um Latossolo Vermelho argiloso de acordo com a tensão de água no solo, uso e manejo. Revista Brasileira de Ciência do Solo, Viçosa - MG, v.27, n.5, p.773-781, 2003.

OLIVEIRA G.C.; SEVERIANO, E.C.; MELLO, C.R. Dinâmica da resistência à penetração de um Latossolo Vermelho da Microrregião de Goiânia-GO. Revista Brasileira de Engenharia Agrícola e Ambiental, Campina Grande, v.11, n.3, p.265-270, 2007.

PENG, X.H.; HORN, R.; ZHANG, B.; ZHAO, Q.G. Mechanisms of soil vulnerability to compaction of homogenized and recompacted Ultisols. Soil \& Tillage Research, Amsterdam, v.76, n.2, p.125-137, 2004.

PAULINO, A.F.; MEDINA, C.C.; AZEVEDO, M.C.B.; SILVEIRA, K.R.P.; TREVISAN, A.A.; MURATA, I.M. Escarificação de um Latossolo Vermelho na pós-colheita de soqueira de cana-de-açúcar. Revista Brasileira de Ciência do Solo, Viçosa - MG, v.28, n.5, p.911-917, 2004.

REICHERT, J.M.; SUZUKI, L.E.A.S.; REINERT, D.J. Compactação do solo em sistemas agropecuários e florestais: identificação, efeitos, limites críticos e mitigação. Tópicos em Ciência do Solo, Viçosa - MG, v.5, p.48-134, 2007. 
RESENDE, M.; CURI, N.; REZENDE, S.B.; CORRÊA, G.F. Pedologia: base para distinção de ambientes. 5.ed. Lavras: UFLA, 2007. 322 p.

SEVERIANO, E.C.; OLIVEIRA, G.C.; DIAS JÚNIOR, M.S.; CASTRO, M.B.; OLIVEIRA, L.F.C.; COSTA, K.A.P. Compactação de solos cultivados com cana-de-açúcar: I - modelagem e quantificação da compactação adicional após as operações de colheita. Engenharia Agrícola, Jaboticabal, v.30, n.3, p.404-413, 2010.

SEVERIANO, E.C.; OLIVEIRA, G.C.; DIAS JÚNIOR, M.S.; OLIVEIRA, L.F.C.; CASTRO, M.B. Pressão de preconsolidação e intervalo hídrico ótimo como indicadores de alterações estruturais do solo em decorrência das operações de colheita da cana-de-açúcar. Revista Brasileira de Ciência do Solo, Viçosa - MG, v.32, n.4, p.1.419-1.427, 2008.

SILVA, V.R.; REINERT, D.J.; REICHERT, J.M.; SOARES, J.M. Fatores controladores da compressibilidade de um Argissolo Vermelho-Amarelo distrófico arênico e de um Latossolo Vermelho distrófico típico. I - Estado inicial de compactação. Revista Brasileira de Ciência do Solo, Viçosa - MG, v.26, n.1, p.1-8, 2002.

SNEDECOR, G.W.; COCHRAN, W.G. Statistical methods. $8^{\text {th }}$ ed. Ames: Iowa State University, 1989. $503 \mathrm{p}$.

SOUZA, Z.M.; PRADO, R.M.; PAIXÃO, A.C.S.; CESARIN, L.G. Sistemas de colheita e manejo da palhada de cana-de-açúcar. Pesquisa Agropecuária Brasileira, Brasília, v.40, n.3, p.271-278, 2005.

SUZUKI, L.E.A.S.; REICHERT, J.M.; REINERT, D.J.; LIMA, C.L.R. Grau de compactação, propriedades físicas e rendimento de culturas em Latossolo e Argissolo. Pesquisa Agropecuária Brasileira, Brasília, v.42, n.8, p.1.159-1.167, 2007.

TORMENA, C.A.; ARAÚJO, M.A.; FIDALSKI, J.; COSTA, J.M. Variação temporal do intervalo hídrico ótimo de um Latossolo Vermelho distroférrico sob sistemas de plantio direto. Revista Brasileira de Ciência do Solo, Viçosa - MG, v.31, n.2, p.211-219, 2007.

ZHUANG, J.; JIN, Y.; MIYAZAKI, T. Estimating water retention characteristic from soil particlesize distribution using a non-similar media concept. Soil Science, Baltimore, v.166, n.5, p.308-321, 2001. 